\title{
Novel insights from non-conserved microRNAs in plants
}

\author{
Zhengrui Qin ${ }^{1}$, Chunlian $\mathrm{Li}^{2}$, Long Mao ${ }^{1 *}$ and Liang $\mathrm{Wu}^{1 *}$ \\ ${ }^{1}$ National Key Facility for Crop Gene Resources and Genetic Improvement, Institute of Crop Science, Chinese Academy of Agricultural Sciences, Beijing, China \\ ${ }^{2}$ State Key Laboratory of Protein and Plant Gene Research, College of Life Sciences, Peking University, Beijing, China
}

Edited by:

Yuhai Cui, Agriculture and Agri-Food

Canada, Canada

\section{Reviewed by:}

Jia-Wei Wang, Shanghai Institute

for Biological Sciences, China

Gang Wu, Zhejiang A\&F University,

China

\section{${ }^{*}$ Correspondence:}

Liang Wu and Long Mao, National

Key Facility for Crop Resources and Genetic Improvement, Institute of

Crop Science, Chinese Academy of

Agricultural Sciences, No. 12

Zhongguancun South Street,

Haidian District, Beijing 100081,

China

e-mail:wuliang@caas.cn;

maolong@caas.cn
Plant microRNAs (miRNAs), a class of small non-coding regulatory RNAs, are canonically 20-24 nucleotides in length and bind to complementary target RNA sequences, guiding target attenuation via mRNA degradation or translation inhibition. Of the annotated miRNA families, evolutionarily conserved families have been well known to extensively regulate analogous targets and play critical roles in plant development and adaptation to adverse environments. By contrast, majority of these families that are merely present in a specific lineage or in a few closely related species have not been well functionally explored until recently. The fast-growing progresses being made in the actions of non-conserved miRNAs nowadays in diverse plant species may represent a highly promising research field in future. This review thereby summarizes the emerging advances in our understanding of the biogenesis, associated effectors, modes to targets, and biological functions of plant non-conserved miRNAs. In addition, it outlines the regulatory units recently discovered between conserved miRNAs and their alternative targets.

Keywords: miRNA, role, secondary siRNA, dicots, monocots

\section{INTRODUCTION}

In the past decade, small silencing RNAs (sRNAs) have been identified as key components of gene modulatory networks in eukaryotes. In plants, based on their origins, structures, and actions on targets, sRNAs are classified into two major categories: small interfering RNAs (siRNAs) and microRNAs (miRNAs; Meyers et al., 2008). Even though the diverse sets of siRNAs, including heterochromatic siRNAs (hc-siRNAs), which are also named repeatassociated siRNAs (ra-siRNAs), trans-acting siRNAs (ta-siRNAs) and natural antisense transcript-derived siRNAs (nat-siRNAs) have similar origination from double-stranded transcripts, their actions on targets are largely dependent on their sizes, for instance, 21-nucleotide (21-nt) siRNAs usually cleave target mRNAs for post-transcriptional gene silencing (PTGS) while 24nt siRNAs guide target chromatin remodeling through a mechanism called RNA-directed DNA methylation (RdDM) mediating transcriptional gene silencing (TGS; Allen and Howell, 2010; Law and Jacobsen, 2010).

Compared with siRNAs, the criteria for defining miRNAs is based on the distinctive nature of miRNA biogenesis. miRNAs come from pre-miRNAs that adopt canonical stem-loop transcripts (Meyers et al., 2008). One important characteristic of miRNA distinct from siRNA is that the sequence of mature miRNA has little heterogeneity from other small RNAs in its hairpin precursor structure. With the powerful capacity of highthroughput sequencing technologies and development of bioinformatics prediction approaches, there has been a dramatic explosion in the number of miRNAs reported in recent years. In most cases, plant miRNAs are 20- to 22-nt in length and mediate target regulation via mRNA digestion or protein translation inhibition. In particular, 24-nt long miRNAs (lmiRNAs) in rice are produced and are able to serve as epigenetic regulators to targets in a DNA methylation manner (Wu et al., 2010), suggesting complicated miRNA populations and function manners in plants.

\section{CONSERVATION OF miRNAs IN PLANTS}

According to the conservation and diversification of miRNAs during evolution in the plant kingdom, miRNA families can be classified to two different categories: the ancient miRNAs and the young miRNAs (Axtell and Bowman, 2008; Tang, 2010; Cuperus et al., 2011). The ancient miRNAs are often highly expressed and evolutionally conserved, while the young miRNAs are expressed lowly or only induced by specific conditions and generally exist only in limited species, resulting in being evolutionarily nonconserved (Cuperus et al., 2011; Taylor et al., 2014).

\section{CONSERVED miRNAs}

Green plants form a clade of eukaryotes that includes green algae, a single-cell organism, and living multicellular organisms, including Cyanophora and Embryophyta. Embryophyta is the most populous subkingdom of green plants formed vegetation on earth, containing mosses, ferns, gymnosperms, and angiosperms (Taylor et al., 2014). Although there are no strictly conserved miRNAs among all organisms with chloroplast because none of the same miRNAs have been identified from single-celled algae Chlamydomonas and multicellular plants (Molnar et al., 2007; Zhao et al., 2007), several ancient miRNAs such as miR156, miR160, miR165/166, miR167, miR319, miR390, miR395, and miR408 are present across land plants as well as in the nonflowering moss, suggesting that these miRNA families are universal in Embryophyta lineages (Taylor et al., 2014). In this review, we designated universal families with identification numbers from 
miR156 to miR408 that are common between gymnosperms and angiosperms including eudicotyledons (dicots) and monocotyledons (monocots) as conserved miRNAs (Cuperus et al., 2011; Taylor et al., 2014). To date, the known biological significance of plant miRNAs are primarily from those conserved miRNAs, which predominantly regulate ancestral transcription factors or physiological enzymes involved in basic plant development or tolerance to stresses.

\section{NON-CONSERVED miRNAs}

A large number of plant miRNAs and their corresponding targets are only present within a few closely related species or appear to be unique to specific species. These lineage-specific miRNAs are customarily called non-conserved miRNAs so as to distinguish them from the conserved miRNAs. Unlike conserved miRNAs with high abundance and low sequence variations, non-conserved miRNAs are often processed imprecisely, expressed weakly, and lack of functional targets; thus in some cases they have been considered as transient products and energy wasters in the plant genome. Despite this, some non-conserved miRNAs are expressed abundantly in specific tissues or greatly induced under particular conditions, supporting a possible physiological role of plant nonconserved miRNAs for special environmental adaptations. Efforts to understand lineage-specific miRNAs in different plants will help elucidate how these young miRNAs affect special growth processes.

\section{BIOGENESIS, ASSOCIATED EFFECTORS, AND ACTIONS OF miRNAs IN PLANTS}

Research on plant miRNA biogenesis and functions was beginning from the highly conserved miRNAs. Genetic conclusions obtained from Arabidopsis and rice firstly have shown that conserved miRNAs share the same machinery in transcription, processing and action fashions. Later on, along with the development of high-throughput sequencing approaches and the resultant produced datasets, people identified more and more lowly expressed and evolutionarily novel miRNAs. Hence, studies on the biogenesis and function pathways of non-conserved miRNAs have been given more and more attentions in recent years.

\section{BIOGENESIS OF CONSERVED MIRNAS}

During long-time evolution history, conserved miRNAs are restricted to similar biogenesis and action machinery. Like protein-coding genes, pri-miRNAs are transcribed by RNA polymerase II (Pol II) and are modified at post-transcriptional layers, occupying a $5^{\prime}$ cap and a $3^{\prime}$ poly (A) tail. But unlike that of in animals, which have diverse origins, plant primary miRNAs are usually transcribed from intergenic regions. The key processor for conserved miRNA precursor and duplex in plant is an RNA endoribonuclease, namely Dicer-like 1 (DCL1), working in a slicing complex comprising double-stranded RNA-binding protein HYPONASTIC LEAVES1 (HYL1), C2H2-zinc finger protein SERRATE (SE), and nuclear cap-binding complex (CBC; Dong et al., 2008; Laubinger et al., 2008). After methylation at the $3^{\prime}$-end by HUA ENHANCER 1 (HEN1; Yu et al., 2005), the mature miRNA duplexes are transported into the cytoplasm from the nucleus by plant exportin 5 ortholog HASTY (Park et al., 2005). Subsequently, one strand called passenger strand is degraded while the other strand called guide stand is recruited into ARGONAUTE1containing RNA-induced silencing complexes (RISCs; Park et al., 2005). These RISCs silence target genes through mRNA degradation or protein translation repression based on RNA-RNA base-pairing (Baumberger and Baulcombe, 2005; Qi et al., 2005; Brodersen et al., 2008).

In addition to sRNA loading and RISCs activities, some AGO proteins such as AGO2 in animal cells have been implicated in increasing mature miRNA levels by feedback mediating miRNA processing and stabilization (Winter and Diederichs, 2011). We are not going to discuss the conserved miRNA pathway in detail here because excellent descriptions on biogenesis and action nature of conserved plant miRNAs have been available in several recent reviews (Voinnet, 2009; Chen, 2012; Rogers and Chen, 2012, 2013; Wu, 2013).

\section{BIOGENESIS OF NON-CONSERVED miRNAs}

Although some non-conserved miRNAs are transcribed from gene exon or intron regions, the majority of them still originate from intergenic loci similar to conserved miRNAs. However, different from those of conserved miRNAs, which occupy multiple copy numbers from several DNA loci, primary transcripts of non-conserved miRNAs are usually transcribed from relatively less genomic regions and are processed by diverse DCLs, thus probably generating some heterogeneous small RNA products in the precursors. Although the major product is miRNA, other processed-sRNAs from the same precursor may also enter alternative RISCs and could be conferred different functions to act on targets (Rajagopalan et al., 2006; Vazquez et al., 2008; Voinnet, 2009).

In contrast with conserved miRNAs, which are characterized to be universally processed by DCL1, the identified non-conserved miRNAs could be produced by distinct DCLs (Figure 1B). Both DCL4 and DCL1 can catalyze the cleavage of RNA transcripts into 21-nt fragments, but DCL4 is originally known to produce endogenous phased and exogenous virus-induced siRNAs (Henderson et al., 2006). Genetic analysis from Arabidopsis and rice has found that several non-conserved miRNAs, especially those with long hairpin precursor structures, were absent from $\mathrm{dcl} 4 \mathrm{but}$ not dcl1 mutants, such as miR822 and miR839 in Arabidopsis and miR7695 in rice, indicating that DCL4 rather than DCL1 is required for these 21-nt non-conserved miRNA biogenesis (Figure 1B; Rajagopalan et al., 2006; Campo et al., 2013).

DCL3 is the only Dicer-like protein that routinely generates 24-nt long sRNA fragments required for hc-siRNA formation in plants. Although most plant miRNAs are 21-nt $(20 \sim 22 \mathrm{nt})$ in length, a class of 24-nt (23-25 nt) lmiRNAs that depend on DCL3 has been recently identified in rice and Arabidopsis (Vazquez et al., 2008; Chellappan et al., 2010; Wu et al., 2010). In contrast to 21-nt miRNAs acting on targets through AGO1-silencing complex at the post-transcriptional level, lmiRNAs are predominantly associated with AGO4 clade proteins which have been originally discovered as essential effectors in RdDM pathway repress targets via DNA methylation (Wu et al., 2010). Interestingly, a subset of pri-miRNAs in rice have been found processed by the coordinated 


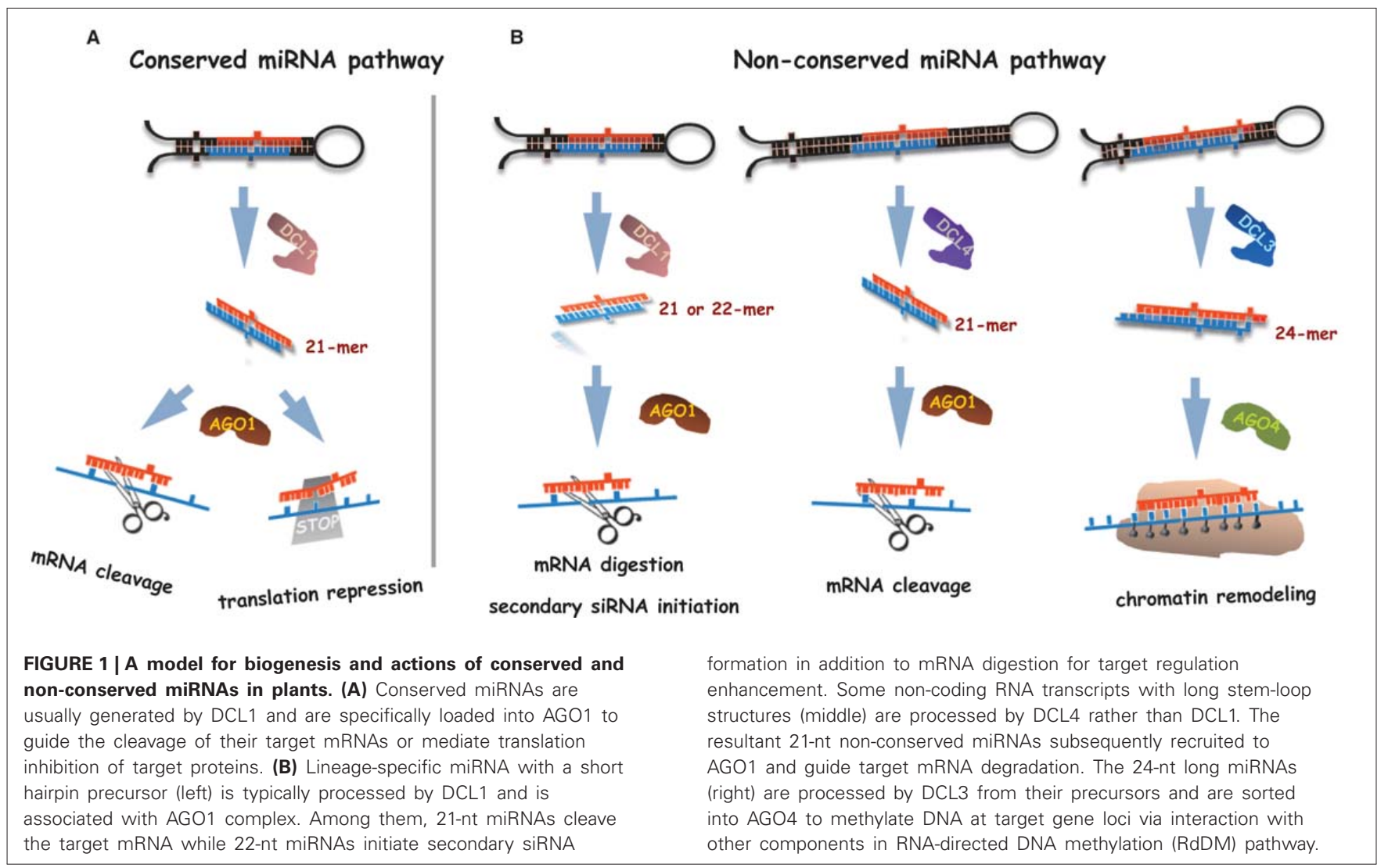

action of DCL1 and DCL3 to simultaneously produce both 21-nt miRNAs and 24-nt lmiRNAs that are hierarchically bound by AGO1 and AGO4 complexes, respectively (Wu et al., 2010). In this regard, a pri-miRNA may have dual functions, producing two miRNA variants that guide either mRNA digestion or chromatin remodeling. Therefore, it is reasonable to think that the 24-nt miRNAs are subjected to short-term selection and evolution, thus improperly entering into the hc-siRNA processing pathway (Figure 1B; Table 1; Wu et al., 2010).

DCL2 is a special DCL protein essential for 22-nt sRNA production. In Arabidopsis dcl3 mutants, the 24-nt long miR825, miR826, and miR827 are replaced by $22-n t$ miRNA species that are not present in wild-type or other $d c l$ mutants, implying a potential role of DCL2 in processing certain pri-miRNAs to generate 22-nt miRNAs (Vazquez et al., 2008).
In summary, the ancient 21-nt miRNAs are generated by DCL1 and recruited in AGO1 effectors, regulating targets by mRNA cleavage or translation inhibition, while non-conserved miRNAs could be processed by any member of the DCL family proteins into diverse-lengths, and hierarchically associated with different AGOs, consequently repressing targets via various modes (Figure 1; Table 1).

\section{miRNA FUNCTIONS IN PLANTS}

Over the last 10 years, the number of annotated miRNAs has increased exponentially through deep sequencing of small RNA transcriptomes from diverse plant species and tissues, but only a few of them have been determined their functional roles. Here we summarize some of recent advances in knowledge on the

Table 1 | Biogenesis and action pathways of conserved and non-conserved miRNAs in plants.

\begin{tabular}{|c|c|c|c|c|c|c|c|}
\hline miRNA type & $\begin{array}{l}\text { Self-complementary } \\
\text { status of precursor }\end{array}$ & Biogenesis & Size (nt) & RISCs & Modes to targets & Example & Reference \\
\hline Conserved & Imperfect & DCL1 & 21 & AGO1 & $\begin{array}{l}\text { mRNA cleavage, } \\
\text { translation inhibition }\end{array}$ & 172,319 & $\begin{array}{l}\text { Palatnik et al. (2003), } \\
\text { Chen (2004) }\end{array}$ \\
\hline \multirow[t]{4}{*}{ Non-conserved } & Less imperfect & DCL1 & 21 & AGO1 & mRNA cleavage & 824,5200 & $\begin{array}{l}\text { Kutter et al. (2007), } \\
\text { Wu et al. (2013) }\end{array}$ \\
\hline & Less imperfect & DCL1 & 22 & AGO1 & $\begin{array}{l}\text { mRNA cleavage, } \\
\text { phased-siRNA initiation }\end{array}$ & 173,6019 & $\begin{array}{l}\text { Montgomery et al. (2008), } \\
\text { Li et al. (2012) }\end{array}$ \\
\hline & Less imperfect & DCL4 & 21 & AGO1 & mRNA cleavage & 822,7695 & $\begin{array}{l}\text { Rajagopalan et al. (2006), } \\
\text { Campo et al. (2013) }\end{array}$ \\
\hline & Less imperfect & DCL3 & 24 & AGO4 & DNA methylation & 1863, 1884 & Wu et al. (2010) \\
\hline
\end{tabular}


associations of miRNAs and physiological processes, particularly focusing on the regulatory effects of the non-conserved miRNAs.

\section{BIOLOGICAL SIGNIFICANCE OF CONSERVED miRNAS}

Features of conserved miRNAs lie not only in their conserved sequences but also in the analogous characteristics of their targets. During long-term evolution, the deeply conserved miRNAs have been integrated early in the regulatory networks to orchestrate plant developmental processes through control of a family of proteins. Despite this, some conserved miRNAs could also evolve lately to acquire novel targets and play alternative roles in specific lineages.

\section{ORIGINAL ROLES OF CONSERVED miRNAs}

As stated above, diversification of conserved miRNA functions in different plants appears to be scarce perhaps owing to the long-term evolutionary selection of old miRNAs and targets (Chapman and Carrington, 2007; Voinnet, 2009; Axtell, 2013). The first evidence of the significance of miRNAs in plant came from two conserved miRNAs, miR319 and miR172 that affect leaf and flower morphogenesis by controlling TEOSINTE BRANCHED1/cycloidea/PCF (TCP) and Apetala2 (AP2) transcription factors (Palatnik et al., 2003; Chen, 2004). From then on, there has been an ever-growing increase in literature on ancient miRNAs targeting versatile transcription factors to influence essential signaling pathways in plant growth and responses to environmental stresses. Due to the space limitations of manuscript required, the conserved biological importance of original miRNAs are not discussed in detail here, and their discussion can be easily found in other frontier reviews (Voinnet, 2009; Rubio-Somoza and Weigel, 2011; Chen, 2012; Poethig, 2013).

\section{ALTERNATIVE ROLES OF CONSERVED miRNAs}

In addition to controlling orthologous targets in various plants, several ancient miRNAs have been recently revealed to recognize non-conserved targets in specific plant species, thus playing alternative roles in plant growth.

Besides targeting growth-regulating factor (GRF) genes in flowering plants, miR396 has been found to affect leaf growth via the acquisition of a neoteric target (bHLH74) in Brassicaceae and Cleomaceae species (Debernardi et al., 2012). Not only GRF family gene mutants, but also loss-of-function of bHLH74 plants displayed abnormal defects in leaf margin and vein pattern formation in Arabidopsis (Debernardi et al., 2012), suggesting spatiotemporal regulation of both ancient and newly acquired targets by miR396 is required for leaf development in Brassicaceae. Intriguingly, the efficiency toward GRFs could be improved by addition of a nucleotide to the miR396 at the expense of reducing activity toward bHLH74 (Debernardi et al., 2012), indicating that the bulges present between miRNA and target pairs may result in differential target modulation effects.

WRKY transcription factors have been clearly known to play a role in plant responses to biotic and abiotic stresses. In sunflower (Helianthus annuus), HaWRKY6 transcript contains a putative target site of miR396. Under high temperature or salicylic acid treatment, sunflower plants show opposite expressions of miR396 and HaWRKY6, suggesting a possible role of its recently evolved regulation by miR396 during early responses to high temperature (Giacomelli et al., 2012). Considering that transformation of sunflower is difficult, the authors used a heterologous system and found that expressing miR396-resistant HaWRKY6 in Arabidopsis not only were phenotypically smaller and accumulated more anthocyanin, but also caused more lethal damage than control plants (Giacomelli et al., 2012), further demonstrating a role of miR396-regulated HaWRKY6 in plants against heat stress. Like the case of miR396-regulated bHLH74 and WRKY, which is not related to canonical target GRF transcription factors in Arabidopsis and sunflower, a non-MYB gene (SGN-U567133) encoding a small nuclear-localized protein with unknown function has been unexpectedly discovered as a novel target for miR159 in tomato (Buxdorf et al., 2010). SGN-U567133 is primarily expressed in flowers may be due to that SlmiR159-mediated the cleavage of this target is remote in this tissues. Moreover, transgenic tomatoes overexpressing miR159-resistant version of SGN-U567133 were observed with strong defects in leaf and flower morphological phenotypes (Buxdorf et al., 2010), suggesting that SlmiR159mediated SGN-U567133 transcript cleavage is critical for tomato development.

The biological consequences reflected above imply that the importance in acquisition of new targets by ancient miRNAs should not be overlooked. Even though a series of non-conserved targets of ancient miRNAs have been predicted by bioinformatics and some cleavage events indeed have been validated by genomewide degradome sequencing efforts (Addo-Quaye et al., 2008; German et al., 2008; Wu et al., 2009b; Li et al., 2010), the biological significance of their interactions is far from known, urgently requiring to be further deciphered in the future.

\section{BIOLOGICAL SIGNIFICANCE OF NON-CONSERVED miRNAS}

Unlike conserved miRNAs, because either abundance of young miRNAs is low or young miRNAs have restricted spatial or temporal expression patterns, they have ever been considered to be evolved from random sequences and conferred few functions. Nonetheless, some non-conserved miRNAs are evident from recent studies that they could also have functions, especially finetuning roles, in target regulatory networks of different plants. Discovery of specific mechanism and functions of non-conserved miRNAs over a large number of conditions has now become a completely fascinating topic of investigation in plant miRNAs.

\section{BIOLOGICAL ROLES OF NON-CONSERVED MIRNAS IN DICOTS}

The first experimental report about non-conserved miRNA functions came from miR824, a Brassicaceae-specific miRNA identified from Arabidopsis. miR824 is formed by the duplication of its unique target gene AGAMOUS-LIKE 16 (AGL16) and mediates AGL16 transcripts digestion at the typical miRNA-cleavage region (Table 2; Kutter et al., 2007). Overexpressing miRNAresistant AGL16 but not wild-type AGL16 significantly increases the density of higher-order stomata in Arabidopsis, suggesting that miR824-mediated AGL16 expression is critical for the development of stomatal complex (Kutter et al., 2007). A recent study also revealed that alteration of AGL16 transcription by interruption of miR824 activity may repress FLOWERING LOCUS T (FT) expression, thereby mediating control of flowering timing 


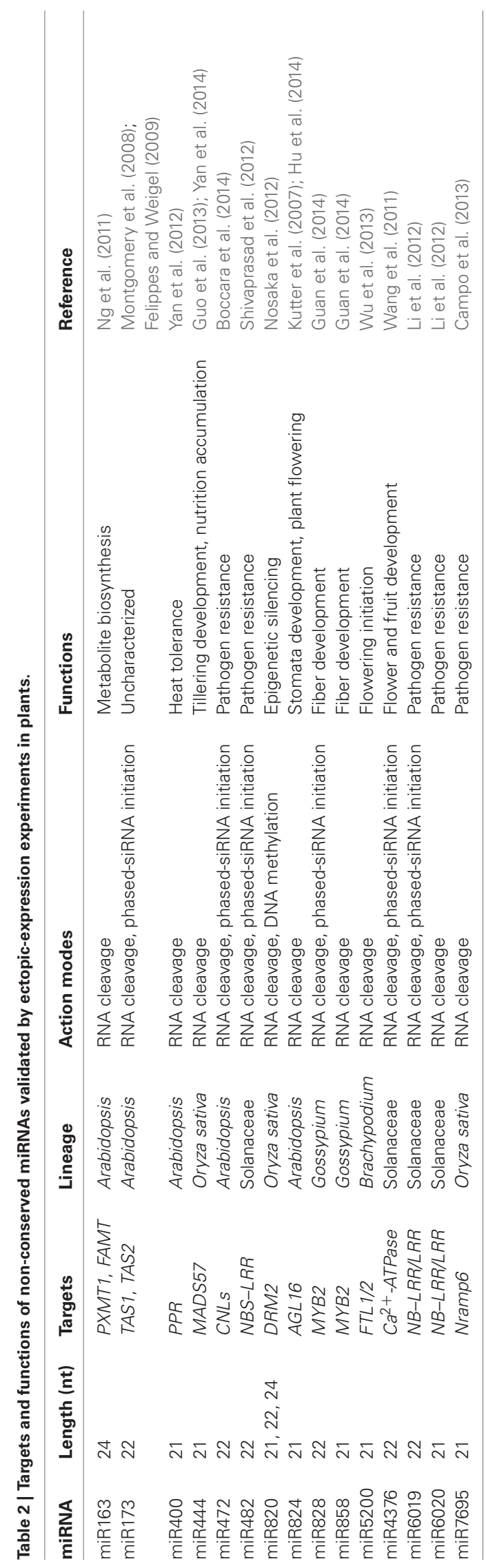

of in Arabidopsis (Hu et al., 2014). These reports suggest that Brassicaceae-specific miR824 is able to play two different fundamental roles during plant growth process. miR163 is a special 24nt miRNA containing a bulge structure between the miRNA and miRNA* in Arabidopsis, and is thus produced by DCL1 rather than DCL3 (Table 2; Wu et al., 2010). miR163 is highly expressed in A. thaliana but is not detectable in A. arenosa and is repressed in resynthesized allotetraploids due to weaker transcriptional expression of miRNA gene as well as lower efficiency of posttranscriptional processing precursors in A. arenosa and resynthesized allotetraploids than in A. thaliana (Ng et al., 2011). Because of being transcribed from inverted repeats, miR163 precursors share high levels of sequence identity with their targets SABATH methyltransferase family protein genes (FAMT and PXMT1). In mir163 mutants, the target expression and plant metabolites profiles are profoundly changed, indicating that miR163-mediated target regulation controls secondary metabolite biosynthesis $(\mathrm{Ng}$ et al., 2011). Notably, although miR163 and its targets can be induced by fungal elicitor alamethicin both in A. arenosa and A. thaliana, the induction level is much different in the two species. This phenomenon suggests that the variation in miR163mediated metabolic pathway may be important for growth vigor and stress adaptation in Arabidopsis species and allopolyploids (Table 2; Ng et al., 2011). It is interesting to explore that whether miR163 activity is also involved in heterosis in other plants besides Arabidopsis.

Another interesting example is miR400, an intronic miRNA, which is co-transcribed with its host gene (At1G32583) in A. thaliana. As a result of heat-induced alternative splicing regulatory machinery, mature miR400 is repressed under heat stress with an increase in its primary transcript levels (Yan et al., 2012). Ectopic miR400 transgenic plants displayed more sensitive to heat stress than wild-type plants, suggesting an essential role of post-transcriptional regulation of miR400 in the acquisition of thermo-tolerance in Arabidopsis (Table 2; Yan et al., 2012). Similar splicing-regulated mechanism is also observed for miR842 and miR846, the two functionally related miRNAs involved in abscisic acid (ABA) response in A. thaliana and A. lyrata (Jia and Rock, 2013). These results implicate a more complex process for generation and regulation of young miRNAs than ancient ones.

The interaction of a 22-nt miRNA with a target mRNA may trigger secondary siRNAs in phase, which is able to amplify the effects of RNA silencing in plants. Moreover, this class of secondary siRNAs may also form non-cell-autonomous signals to silence other genes in trans. Consequently, it is reasonable that guiding target transcripts to generate secondary siRNAs appears to be an efficient approach for the enhancement of target mRNA degradation by miRNAs. miR173, a typical 22-nt miRNA arisen from fold-back precursors with asymmetric bulges, targets a noncoding RNA and has been characterized as an initiator of phased siRNA formation at TAS1 and TAS2 loci in A. thaliana and A. lyrata (Table 2; Chen et al., 2007, 2010; Montgomery et al., 2008; Felippes and Weigel, 2009; Cuperus et al., 2010; Wu et al., 2012; Yoshikawa et al., 2013). Although pentatricopeptide repeat (PPR) transcripts have been experimentally validated to be digested by miR173-triggered phased siRNAs for long time, the physiological effects of miR173 and miR173-triggered phased siRNAs are still 
unknown (Allen et al., 2005; Montgomery et al., 2008; Wu et al., 2012).

The first biological relevance of interaction between nonconserved miRNA and target through secondary siRNA signaling has been discovered in Solanaceae plants (Wang et al., 2011). Subject to post-transcriptional processing modulation, miR4376 randomly occurs in Solanaceae families and mediates auto-inhibited $\mathrm{Ca}^{2+}$-ATPase (ACA10) mRNA degradation and phased secondary siRNA formation from target transcripts (Table 2; Wang et al., 2011). In tomato, low abundance of ACA10-originated phased siRNAs is directly correlated with the limited accumulation of miR4376 in flowers and fruits, whereas high-expression of miR4376 in leaves leads to a decrease of ACA10 with an increase in phased siRNA accumulation. Ectopic expression of ACA10 with a mutated miR4376 target site in tomato specifically altered the flower morphology and fruit yield, supporting the significance of miR4376-mediated ACA10 control in plant reproduction (Table 2; Wang et al., 2011).

When invaded by pathogens or insects, plants may change some endogenous gene expressions including miRNA accumulations to trigger resistance responses. Tomato miR482 and tobacco miR6019 are such representative species-specific miRNAs in immune response to pathogens (Li et al., 2012; Shivaprasad et al., 2012). As a member of 22-nt miRNA family, miR482 slices the mRNAs of nucleotide-binding site-leucine-rich repeat (NBS-LRR) disease resistance proteins with coiled-coil (CC) domains at their N-terminus, and subsequently triggers a class of secondary phased siRNAs dependent on RNA-dependent RNA polymerase 6 (RDR6) activities. These secondary siRNAs not only target their precursors but also act on other mRNAs of defense-related proteins, resulting in a regulatory cascade formed in plant defense systems (Table 2; Shivaprasad et al., 2012). If tomatoes are challenged with viruses or bacteria, miR482mediated target mRNA decay and secondary siRNA production are able to be inhibited, and thereby a large number of NBSLRR proteins are increased, directly giving rise to a defense response against pathogen attack (Shivaprasad et al., 2012). In tobacco, miR6019 cleaves Toll-interleukin-1 receptor-nucleotide binding-LRR (TIR-NB-LRR) immune receptor $N$ gene transcripts and guides synthesis of 21-nt secondary siRNAs requiring on RDR6- and DCL4 activities in phase with the target site (Li et al., 2012). Transient expression of N-targeting miR6019 in Nicotiana benthamiana attenuates N-mediated resistance to tobacco mosaic virus (TMV), indicating that tobacco must block miR6019-mediated attenuation of $R$ gene expression to avoid biotic incursion (Table 2; Li et al., 2012). Interestingly, another 21-nt miRNA, namely miR6020, also mediates $N$ transcripts degradation and resistance to TMV, but it is unable to induce secondary siRNAs attributed to the absence of asymmetric bulge in its precursor structures (Table 2; Li et al., 2012; Manavella et al., 2012; Fei et al., 2013). These results indicate the involvement of two parts of complicated non-conserved miRNAmediated silencing systems for precise regulation of $R$ gene expressions in counteraction of pathogen attack in Solanaceae plants.

In addition to perceiving specific pathogen effectors, the nonconserved miRNAs-mediated secondary siRNAs have also been explored their roles in signaling events triggered by pathogenassociated molecular patterns (PAMPs). In transgenic plants lacking miR472, an Arabidopsis-specific miRNA, recapitulates the basal immunity phenotype of $r d r 6$ mutant which exhibits enhanced basal resistance toward a virulent Pseudomonas syringae strain, suggesting that miR472 and RDR6-mediated CC-NBLRRs (CNLs) silencing pathway may have checkpoints modulating PAMP-triggered immunity (PTI; Boccara et al., 2014). Furthermore, overexpression of miR472 could enhance the number of secondary siRNAs at CNLs and compromise the PTI response when plants are subject to a biotic environment, supporting an essential role of secondary siRNAs by miR472 in CNL modulation and disease resistance (Table 2; Boccara et al., 2014). Because excessive $R$ gene multiplication may lose high fitness costs (Heil and Baldwin, 2002), these non-conserved miRNA-mediated repression of $R$ gene amplification and diversification would be of great biological significance in plants benefiting substantial growth fitness and defense.

The phased siRNAs generated by non-conserved miRNA also participate in plant development. GLABROUS1 (GL1) is a member of R2R3 MYB family of transcription factor and promotes leaf trichome development in Arabidopsis (Pesch and Hulskamp, 2009). There are two GL1 genes including GhMYB2A and GhMYB2D homeologs in cotton, an allotetraploid (Gossypium hirsutum, AADD). Intriguingly, both of two MYB2 transcripts could be targeted by miR828 and miR858 in G. hirsutum, but more secondary siRNAs could be produced from GhMYB2D loci than that from GhMYB2A, since the former gene expressed much higher than the latter during fiber initiation (Table 2; Guan et al., 2014).The authors found that only overexpressing natural GhMYB2A but not GhMYB2D complements the $g l 1$ phenotype, but if mutate the miR828-binding site or replace the downstream target sequence in $G h M Y B 2 D$, it can also restore trichome development in gll mutants because the ta-siRNA production are abolished from GhMYB2D transcripts. This observation thus illustrates a unique role of mirR828 and mir858 in functional divergence between target homeologous genes for cotton fiber development (Guan et al., 2014).

Above all, it is apparent that non-conserved miRNAs have diverse functions in multiple biological processes in dicotyledons. With more investigation of miRNAs from distinct lineages, more physiological implications of non-conserved miRNAs will be uncovered from dicots in the future.

\section{BIOLOGICAL ROLES OF NON-CONSERVED MIRNAS IN MONOCOTS}

Compared with non-conserved miRNA roles characterized from dicots, those from monocots are even more limited.

miR444, which targets a class of MADS transcription factors in rice plants, has been identified for nearly 10 years (Sunkar et al., 2005). Although miR444 is abundantly accumulated in diverse monocots, its biological roles are obscure until two exciting discoveries made recently (Wu et al., 2009b). On the one hand, miR444 modulates OsMADS57, the product of which interacts with a TCP-family transcription factor (TEOSINTE BRANCHED1, OsTB1), and acts on Dwarf14 (D14), the potential receptor of strigolactones to control the outgrowth of axillary buds and tillering in rice (Table 2; 
Guo et al., 2013). On the other hand, miR444 could simultaneously control four MADS-box genes, and may participate in $\mathrm{NO}_{3}^{-}$and $\mathrm{Pi}^{-}$signaling pathways through alteration of lateral root architecture when rice are subject to detrimental nutrient environments (Yan et al., 2014). These findings suggest miR444 is able to play multiple roles in rice growth, and perhaps more additional roles of miR444 could be identified from further explorations in other monocots in addition to rice.

Excessive transposable elements (TEs) can destroy a eukaryotic genome; many organisms thus have developed diverse mechanisms to inhibit TE activities. Majority of transposons and repeat elements in plants have been clearly known to be silenced by the RdDM machinery; however, how the components in the RdDM pathway are regulated remains poorly understood. miR820 is a species of 21-, 22-, and 24-nt small RNAs derived from a class of CACTA DNA transposon transcripts, potentially targeting a de novo DNA methyltransferase gene DRM2 at both transcriptional and post-transcriptional levels (Table 2; Wu et al., 2010; Nosaka et al., 2012). The ectopic expression level of the OsDRM2:GFP fusion gene with an intact miR820 recognition site is observed much lower than that of genes with synonymous mutations, indicating that DRM2 is indeed negatively regulated by miR 820 in rice (Nosaka et al., 2012). Nevertheless, there is no clear inverse expression relationship between the levels of miR820 and targets, suggesting that miR820 may only reduce the amount of DRM2 expression but may not abolish it completely (Nosaka et al., 2012). In transgenic plants showing DRM2 RNAi and premiR820 overexpression, several transposons are de novo activated due to the decrease of DNA methylation status in cis (Nosaka et al., 2012). Together with the observation that the nucleotide sequence of miR820 and its recognition site within the target gene is co-evolved, these findings demonstrate that miR820-mediated DRM2 modulation is essential for the maintenance of low transposon activities in rice (Nosaka et al., 2012). Future work will be needed to dissect miR820 expression pattern to define when and where rice accumulates more miR820 to enable transposon released.

Monocots have 24-nt secondary siRNAs in addition to 21-nt siRNAs. In rice, miR2118 and miR2275 are involved in initiation of these two classes of siRNAs, respectively (Song et al., 2012). Intriguingly, both miR2118 and miR2275 as well as the 21- and 24-nt secondary siRNAs have been found preferentially expressed in panicle and stamens, suggesting that these sRNAs may functionally contribute to reproductive organ development in rice (Song et al., 2012). Nevertheless, experimental genetic studies by overproducing and silencing miR2118 and miR2275 activities are required to further clarify their respective roles in rice panicle and stamens formation.

Through high-throughput sequencing, Campo et al. (2013) identified a novel DCL4-dependent miRNA designated miR7695 from blast fungus-treated rice plants. This miRNA targets an alternatively spliced transcript of Nramp6 (natural resistanceassociated macrophage protein 6; Table 2; Campo et al., 2013). Compared with non-transformed plants, the percentage of M. oryzae diseased leaf area exhibited much lower than that in miR7695 overexpressing transgenic plants, suggesting that
miR7695 contributes to plant resistance to fungal pathogen (Table 2; Campo et al., 2013). Interestingly, miR7695 can only mediate cleavage of one Nramp6 splicing variant but not of all transcripts, representing a complicated regulatory machinery of plant immunity requiring in miRNA-mediated gene regulation (Campo et al., 2013).

In addition to rice, the physiological connections between non-conserved miRNAs and plant growth were also investigated in other monocots. With the attributes of small stature, short generation time, and small genome, Brachypodium is an ideal biological research model for temperate cereals such as wheat and barley, and has been implicated in occupying special genetic pathways for flowering time control compared with eudicotyledons and rice (Higgins et al., 2010). Although numerous lineage-specific miRNAs has been identified from Brachypodium (Wei et al., 2009; Zhang et al., 2009; Jeong et al., 2013), none of their roles are clear until a recent study by us. We characterized a Pooideae-specific miRNA (miR5200) targeting two FLOWERING LOCUS $T$ orthologous genes in Brachypodium in a mRNA cleavage manner. miR5200 has similar tissue expression patterns with BdFTL1 and BdFTL2 mRNAs that distinctively accumulate in plant leaves but are absent in shoot apices, thus spatially conferring regulatory activity to the targets (Table 2; $\mathrm{Wu}$ et al., 2013). Ectopic expression of miR5200 in transgenic Brachypodium plants significantly decreases BdFTL1 and BdFTL2 as well as downstream gene expressions and thereby severely delays flowering time. Furthermore, miR5200 is sensitive to photoperiod, exhibiting highly accumulated when Brachypodium plants are grown in short-day (SD) conditions but dramatically repressed in plants grown under long-day (LD) environments. Interestingly, we found that the histone status of $\mathrm{H} 3 \mathrm{~K} 27 \mathrm{me} 3$ and H3K4me3 in miR5200 precursor genes could change according to day-length, resulting in distinct expression of miR5200 for photoperiodic control of flowering under SD and LD conditions (Wu et al., 2013). Blocking of miR5200 activity in plants through a targeted mimic approach specifically alters flowering time in SD conditions but not in LD conditions, indicating that miR5200-mediated regulation of FT-like gene plays an important role in flowering initiation in Brachypodium (Table 2; Wu et al., 2013). Because photoperiodic regulation of miR5200 appears to be prevalent in Pooideae plants (Wu et al., 2013), it may be feasible to artificially manipulate miR5200 behavior to improve agricultural traits of wheat and barley for wider adaptations in the future.

\section{CONCLUSION AND REMARKS}

The extraordinarily fast development of high-throughput sequencing technologies has enabled scientists to uncover a large number of novel sRNAs in recent days. Countless bioinformatics data of miRNAs have been released and become freely available in updated miRNA databases, providing significant basis for miRNA functional investigations. However, compared to conserved miRNAs whose functions were extensively explored and validated, non-conserved and lineage-specific miRNAs have been ignored in biological studies even if they account for the major part of miRNA families. 
Encouragingly, growing bodies of evidence continuously illustrate that non-conserved miRNAs are also regulators of gene expression and sometimes function as crucial determinants of plant morphology as well as defense against pathogen disease (Zhai et al., 2011). Unlike ancient miRNAs, which usually serve as transcription factor modulators, non-conserved miRNAs have broader predicted targets, including active enzymes and physiological proteins involved in adaption to multifarious conditions.

Besides of guiding gene transcript digestion and protein translation inhibition, non-conserved miRNAs can control targets through inducing secondary siRNA formation or trigging DNA methylation. miR173 in Arabidopsis, miR2275 in rice, and miR482 in tomatoes are typical examples of non-conserved miRNAs that mediate phased siRNA initiation for the enhancement of target regulation (Shivaprasad et al., 2012; Song et al., 2012; Fei et al., 2013). Although it is clear that lmiRNAs act on target DNA in rice, their biological role and phenotypic relevance in epigenetic modeling have not yet been illustrated (Wu et al., 2010).

Moreover, regulatory modules composed by multiple conserved miRNAs and targets have been identified to be involved in diverse interconnected biological programs, including flowering maturation, flowering initiation, lateral organ growth, nodule development, senescence, and phytohormone signaling (Wu et al., 2009a; Koyama et al., 2010; Gutierrez et al., 2012; Mao et al., 2013; Rubio-Somoza and Weigel, 2013; Curaba et al., 2014; Spanudakis and Jackson, 2014), implying that there also may be conceivably coordinated relationships between conserved and non-conserved miRNAs in diverse genetic pathways (Jin et al., 2013). For instance, whether miR156 and miR172 affect miR5200-controlled FT module in temperate cereals, whether miR159-regulated MYB transcription factors can influence miR842 and miR846 expressions in Arabidopsis plants subject to ABA, whether miR156SPLs module is able to upstreamly affect primary miR444 transcripts to control tillering in rice, and whether miR393 affects the accumulation of defense miRNAs required for secondary siRNA generation in biotic-stress responses through auxin signaling pathway are interesting to be investigated. Thus, it is important to explore and characterize more possible mechanistic significances of non-conserved miRNAs at first in different plant species.

Genetic engineering is thought to be the most rapid way to resolve the challenges in modern agriculture nowadays. Because plenty of miRNAs have been found to be involved in plant morphogenesis, researchers are beginning to take advantage of operating miRNA actions to improve plant architecture for increasing crop yield. miR156-modulated SPL14 has been implicated in the semi-dominant quantitative trait locus termed as IPA1 in rice (Jiao et al., 2010; Miura et al., 2010). Introduction of a point mutation in SPL14 could interrupt miR156-mediated regulation of SPL14 and generated an optimized rice plant with few unproductive branches, enhanced lodging resistance, and improved grain yields (Jiao et al., 2010). Laccase-like proteins (LACs) are regulated by miR397 and are involved in brassinosteroids (BR) signaling. Overexpression of miR397 in rice dramatically represses $L A C$ expressions, simultaneously enlarges grain size, and promotes panicle branching, thus significantly increasing grain production (Zhang et al., 2013). Now that increasing rice yield through miRNA actions is practical, it will be promising to improve agricultural traits of crops more economically by finetuning the regulatory module between non-conserved miRNA and targets in the future.

\section{ACKNOWLEDGMENTS}

Our work was supported by the National Natural Science Foundation of China (\#31200181), the Transgenic Key Project of MOA (2013ZX08009-001), the National Basic Research "973" (\#2010CB125902), and a core research budget from a non-profit governmental research institution (ICS, CAAS). We apologize to the authors whose research is not cited in this review because of space limitations.

\section{REFERENCES}

Addo-Quaye, C., Eshoo, T. W., Bartel, D. P., and Axtell, M. J. (2008). Endogenous siRNA and miRNA targets identified by sequencing of the Arabidopsis degradome. Curr. Biol. 18, 758-762. doi: 10.1016/j.cub.2008.04.042

Allen, E., and Howell, M. D. (2010). miRNAs in the biogenesis of transacting siRNAs in higher plants. Semin. Cell Dev. Biol. 21, 798-804. doi: 10.1016/j.semcdb.2010.03.008

Allen, E., Xie, Z., Gustafson, A. M., and Carrington, J. C. (2005). microRNAdirected phasing during trans-acting siRNA biogenesis in plants. Cell 121, 207221. doi: 10.1016/j.cell.2005.04.004

Axtell, M. J. (2013). Classification and comparison of small RNAs from plants. Annu. Rev. Plant Biol. 64, 137-159. doi: 10.1146/annurev-arplant-050312120043

Axtell, M. J., and Bowman, J. L. (2008). Evolution of plant microRNAs and their targets. Trends Plant Sci. 13, 343-349. doi: 10.1016/j.tplants.2008.03.009

Baumberger, N., and Baulcombe, D. C. (2005). Arabidopsis ARGONAUTE1 is an RNA Slicer that selectively recruits microRNAs and short interfering RNAs. Proc. Natl. Acad. Sci. U.S.A. 102, 11928-11933. doi: 10.1073/pnas.0505461102

Boccara, M., Sarazin, A., Thiebeauld, O., Jay, F., Voinnet, O., Navarro, L., et al. (2014). The Arabidopsis miR472-RDR6 silencing pathway modulates PAMP- and effector-triggered immunity through the post-transcriptional control of disease resistance genes. PLoS Pathog. 10:e1003883. doi: 10.1371/journal.ppat.1003883

Brodersen, P., Sakvarelidze-Achard, L., Bruun-Rasmussen, M., Dunoyer, P., Yamamoto, Y. Y., Sieburth, L., et al. (2008). Widespread translational inhibition by plant miRNAs and siRNAs. Science 320, 1185-1190. doi: 10.1126/science. 1159151

Buxdorf, K., Hendelman, A., Stav, R., Lapidot, M., Ori, N., and Arazi, T. (2010). Identification and characterization of a novel miR159 target not related to MYB in tomato. Planta 232, 1009-1022. doi: 10.1007/s00425-010-1231-9

Campo, S., Peris-Peris, C., Sire, C., Moreno, A. B., Donaire, L., Zytnicki, M., et al. (2013). Identification of a novel microRNA (miRNA) from rice that targets an alternatively spliced transcript of the Nramp6 (Natural resistance-associated macrophage protein 6) gene involved in pathogen resistance. New Phytol. 199, 212-227. doi: 10.1111/nph.12292

Chapman, E. J., and Carrington, J. C. (2007). Specialization and evolution of endogenous small RNA pathways. Nat. Rev. Genet. 8, 884-896. doi: $10.1038 / \mathrm{nrg} 2179$

Chellappan, P., Xia, J., Zhou, X., Gao, S., Zhang, X., Coutino, G., et al. (2010). siRNAs from miRNA sites mediate DNA methylation of target genes. Nucleic Acids Res. 38, 6883-6894. doi: 10.1093/nar/gkq590

Chen, H. M., Chen, L. T., Patel, K., Li, Y. H., Baulcombe, D. C., and Wu, S. H. (2010). 22-Nucleotide RNAs trigger secondary siRNA biogenesis in plants. Proc. Natl. Acad. Sci. U.S.A. 107, 15269-15274. doi: 10.1073/pnas.1001738107

Chen, H. M., Li, Y. H., and Wu, S. H. (2007). Bioinformatic prediction and experimental validation of a microRNA-directed tandem trans-acting siRNA cascade in Arabidopsis. Proc. Natl. Acad. Sci. U.S.A. 104, 3318-3323. doi: 10.1073/pnas.0611119104

Chen, X. (2004). A microRNA as a translational repressor of APETALA2 in Arabidopsis flower development. Science 303, 2022-2025. doi: 10.1126/science. 1088060 
Chen, X. (2012). Small RNAs in development-insights from plants. Curr. Opin. Genet. Dev. 22, 361-367. doi: 10.1016/j.gde.2012.04.004

Cuperus, J. T., Carbonell, A., Fahlgren, N., Garcia-Ruiz, H., Burke, R. T., Takeda, A., et al. (2010). Unique functionality of 22-nt miRNAs in triggering RDR6dependent siRNA biogenesis from target transcripts in Arabidopsis. Nat. Struct. Mol. Biol. 17, 997-1003. doi: 10.1038/nsmb.1866

Cuperus, J. T., Fahlgren, N., and Carrington, J. C. (2011). Evolution and functional diversification of MIRNA genes. Plant Cell 23, 431-442. doi: $10.1105 /$ tpc. 110.082784

Curaba, J., Singh, M. B., and Bhalla, P. L. (2014). miRNAs in the crosstalk between phytohormone signalling pathways. J. Exp. Bot. 65, 1425-1438. doi: 10.1093/jxb/eru002

Debernardi, J. M., Rodriguez, R. E., Mecchia, M. A., and Palatnik, J. F. (2012). Functional specialization of the plant miR396 regulatory network through distinct microRNA-target interactions. PLoS Genet. 8:e1002419. doi: 10.1371/journal.pgen.1002419

Dong, Z., Han, M. H., and Fedoroff, N. (2008). The RNA-binding proteins HYL1 and SE promote accurate in vitro processing of pri-miRNA by DCL1. Proc. Natl. Acad. Sci. U.S.A. 105, 9970-9975. doi: 10.1073/pnas.0803356105

Fei, Q., Xia, R., and Meyers, B. C. (2013). Phased, secondary, small interfering RNAs in posttranscriptional regulatory networks. Plant Cell 25, 2400-2415. doi: 10.1105/tpc.113.114652

Felippes, F. F., and Weigel, D. (2009). Triggering the formation of tasiRNAs in Arabidopsis thaliana: the role of microRNA miR173. EMBO Rep. 10, 264-270. doi: 10.1038/embor.2008.247

German, M. A., Pillay, M., Jeong, D. H., Hetawal, A., Luo, S., Janardhanan, P., et al. (2008). Global identification of microRNA-target RNA pairs by parallel analysis of RNA ends. Nat. Biotechnol. 26, 941-946. doi: 10.1038/nbt1417

Giacomelli, J. I., Weigel, D., Chan, R. L., and Manavella, P. A. (2012). Role of recently evolved miRNA regulation of sunflower HaWRKY6 in response to temperature damage. New Phytol. 195, 766-773. doi: 10.1111/j.14698137.2012.04259.x

Guan, X., Pang, M., Nah, G., Shi, X., Ye, W., Stelly, D. M., et al. (2014). miR828 and miR858 regulate homoeologous MYB2 gene functions in Arabidopsis trichome and cotton fibre development. Nat. Commun. 5, 3050. doi: 10.1038/ncomms4050

Guo, S., Xu, Y., Liu, H., Mao, Z., Zhang, C., Ma, Y., et al. (2013). The interaction between OsMADS57 and OsTB1 modulates rice tillering via DWARF14. Nat. Commun. 4, 1566. doi: 10.1038/ncomms 2542

Gutierrez, L., Mongelard, G., Flokova, K., Pacurar, D. I., Novak, O., Staswick, P., et al. (2012). Auxin controls Arabidopsis adventitious root initiation by regulating jasmonic acid homeostasis. Plant Cell 24, 2515-2527. doi: 10.1105/tpc.112.099119

Heil, M., and Baldwin, I. T. (2002). Fitness costs of induced resistance: emerging experimental support for a slippery concept. Trends Plant Sci. 7, 61-67. doi: 10.1016/S1360-1385(01)02186-0

Henderson, I. R., Zhang, X., Lu, C., Johnson, L., Meyers, B. C., Green, P. J., et al. (2006). Dissecting Arabidopsis thaliana DICER function in small RNA processing, gene silencing and DNA methylation patterning. Nat. Genet. 38, 721-725. doi: 10.1038/ng1804

Higgins, J. A., Bailey, P. C., and Laurie, D. A. (2010). Comparative genomics of flowering time pathways using Brachypodium distachyon as a model for the temperate grasses. PLOS ONE 5:e10065. doi: 10.1371/journal.pone. 0010065

Hu, J. Y., Zhou, Y., He, F., Dong, X., Liu, L. Y., Coupland, G., et al. (2014). miR824-regulated AGAMOUS-LIKE16 contributes to flowering time repression in Arabidopsis. Plant cell 26, 2024-2037. doi: 10.1105/tpc.114. 124685

Jeong, D. H., Schmidt, S. A., Rymarquis, L. A., Park, S., Ganssmann, M., German, M. A., et al. (2013). Parallel analysis of RNA ends enhances global investigation of microRNAs and target RNAs of Brachypodium distachyon. Genome Biol. 14, R145. doi: 10.1186/gb-2013-14-12-r145

Jia, F., and Rock, C. D. (2013). MIR846 and MIR842 comprise a cistronic MIRNA pair that is regulated by abscisic acid by alternative splicing in roots of Arabidopsis. Plant Mol. Biol. 81, 447-460. doi: 10.1007/s11103-013-0015-6

Jiao, Y., Wang, Y., Xue, D., Wang, J., Yan, M., Liu, G., et al. (2010). Regulation of OsSPL14 by OsmiR156 defines ideal plant architecture in rice. Nat. Genet. 42, 541-544. doi: 10.1038/ng.591
Jin, D., Wang, Y., Zhao, Y., and Chen, M. (2013). MicroRNAs and their cross-talks in plant development. J. Genet. Genomics 40, 161-170. doi: 10.1016/j.jgg.2013.02.003

Koyama, T., Mitsuda, N., Seki, M., Shinozaki, K., and Ohme-Takagi, M. (2010). TCP transcription factors regulate the activities of ASYMMETRIC LEAVES1 and miR164, as well as the auxin response, during differentiation of leaves in Arabidopsis. Plant Cell 22, 3574-3588. doi: 10.1105/tpc.110. 075598

Kutter, C., Schob, H., Stadler, M., Meins, F. Jr., and Si-Ammour, A. (2007). MicroRNA-mediated regulation of stomatal development in Arabidopsis. Plant Cell 19, 2417-2429. doi: 10.1105/tpc.107.050377

Laubinger, S., Sachsenberg, T., Zeller, G., Busch, W., Lohmann, J. U., Ratsch, G., et al. (2008). Dual roles of the nuclear cap-binding complex and SERRATE in pre-mRNA splicing and microRNA processing in Arabidopsis thaliana. Proc. Natl. Acad. Sci. U.S.A. 105, 8795-8800. doi: 10.1073/pnas.0802493105

Law, J. A., and Jacobsen, S. E. (2010). Establishing, maintaining and modifying DNA methylation patterns in plants and animals. Nat. Rev. Genet. 11, 204-220. doi: $10.1038 / \operatorname{nrg} 2719$

Li, F., Pignatta, D., Bendix, C., Brunkard, J. O., Cohn, M. M., Tung, J., et al. (2012). MicroRNA regulation of plant innate immune receptors. Proc. Natl. Acad. Sci. U.S.A. 109, 1790-1795. doi: 10.1073/pnas.1118282109

Li, Y. F., Zheng, Y., Addo-Quaye, C., Zhang, L., Saini, A., Jagadeeswaran, G., et al. (2010). Transcriptome-wide identification of microRNA targets in rice. Plant J. 62, 742-759. doi: 10.1111/j.1365-313X.2010.04187.x

Manavella, P. A., Koenig, D., and Weigel, D. (2012). Plant secondary siRNA production determined by microRNA-duplex structure. Proc. Natl. Acad. Sci. U.S.A. 109, 2461-2466. doi: 10.1073/pnas.1200169109

Mao, G., Turner, M., Yu, O., and Subramanian, S. (2013). miR393 and miR164 influence indeterminate but not determinate nodule development. Plant Signal. Behav. 8:e26753. doi: 10.4161/psb.26753

Meyers, B. C., Axtell, M. J., Bartel, B., Bartel, D. P., Baulcombe, D., Bowman, J. L., et al. (2008). Criteria for annotation of plant microRNAs. Plant Cell 20, 31863190. doi: $10.1105 /$ tpc. 108.064311

Miura, K., Ikeda, M., Matsubara, A., Song, X. J., Ito, M., Asano, K., et al. (2010). OsSPL14 promotes panicle branching and higher grain productivity in rice. Nat. Genet. 42, 545-549. doi: 10.1038/ng.592

Molnar, A., Schwach, F., Studholme, D. J., Thuenemann, E. C., and Baulcombe, D. C. (2007). miRNAs control gene expression in the single-cell alga Chlamydomonas reinhardtii. Nature 447, 1126-1129. doi: 10.1038/nature05903

Montgomery, T. A., Yoo, S. J., Fahlgren, N., Gilbert, S. D., Howell, M. D., Sullivan, C. M., et al. (2008). AGO1-miR173 complex initiates phased siRNA formation in plants. Proc. Natl. Acad. Sci. U.S.A. 105, 20055-20062. doi: 10.1073/pnas.0810241105

Ng, D. W., Zhang, C., Miller, M., Palmer, G., Whiteley, M., Tholl, D., et al. (2011). cis- and trans-Regulation of miR163 and target genes confers natural variation of secondary metabolites in two Arabidopsis species and their allopolyploids. Plant Cell 23, 1729-1740. doi: 10.1105/tpc.111.083915

Nosaka, M., Itoh, J., Nagato, Y., Ono, A., Ishiwata, A., and Sato, Y. (2012). Role of transposon-derived small RNAs in the interplay between genomes and parasitic DNA in rice. PLoS Genet. 8:e1002953. doi: 10.1371/journal.pgen. 1002953

Palatnik, J. F., Allen, E., Wu, X., Schommer, C., Schwab, R., Carrington, J. C., et al. (2003). Control of leaf morphogenesis by microRNAs. Nature 425, 257-263. doi: 10.1038/nature01958

Park, M. Y., Wu, G., Gonzalez-Sulser, A., Vaucheret, H., and Poethig, R. S. (2005). Nuclear processing and export of microRNAs in Arabidopsis. Proc. Natl. Acad. Sci. U.S.A. 102, 3691-3696. doi: 10.1073/pnas.0405570102

Pesch, M., and Hulskamp, M. (2009). One, two, three...models for trichome patterning in Arabidopsis? Curr. Opin. Plant Biol. 12, 587-592. doi: 10.1016/j.pbi.2009.07.015

Poethig, R. S. (2013). Vegetative phase change and shoot maturation in plants. Curr. Top. Dev. Biol. 105, 125-152. doi: 10.1016/B978-0-12-396968-2.00005-1

Qi, Y., Denli, A. M., and Hannon, G. J. (2005). Biochemical specialization within Arabidopsis RNA silencing pathways. Mol. Cell 19, 421-428. doi: 10.1016/j.molcel.2005.06.014

Rajagopalan, R., Vaucheret, H., Trejo, J., and Bartel, D. P. (2006). A diverse and evolutionarily fluid set of microRNAs in Arabidopsis thaliana. Genes Dev. 20, 3407-3425. doi: 10.1101/gad.1476406 
Rogers, K., and Chen, X. (2012). microRNA biogenesis and turnover in plants. Cold. Spring Harb. Symp. Quant. Biol. 77, 183-194. doi: 10.1101/sqb.2013.77.014530

Rogers, K., and Chen, X. (2013). Biogenesis, turnover, and mode of action of plant microRNAs. Plant Cell 25, 2383-2399. doi: 10.1105/tpc.113.113159

Rubio-Somoza, I., and Weigel, D. (2011). MicroRNA networks and developmental plasticity in plants. Trends Plant Sci. 16, 258-264. doi: 10.1016/j.tplants.2011.03.001

Rubio-Somoza, I., and Weigel, D. (2013). Coordination of flower maturation by a regulatory circuit of three microRNAs. PLoS Genet. 9:e1003374. doi: 10.1371/journal.pgen.1003374

Shivaprasad, P. V., Chen, H. M., Patel, K., Bond, D. M., Santos, B. A., and Baulcombe, D. C. (2012). A microRNA superfamily regulates nucleotide binding site-leucine-rich repeats and other mRNAs. Plant Cell 24, 859-874. doi: 10.1105/tpc.111.095380

Song, X., Li, P., Zhai, J., Zhou, M., Ma, L., Liu, B., et al. (2012). Roles of DCL4 and DCL3b in rice phased small RNA biogenesis. Plant J. 69, 462-474. doi: 10.1111/j.1365-313X.2011.04805.x

Spanudakis, E., and Jackson, S. (2014). The role of microRNAs in the control of flowering time. J. Exp. Bot. 65, 365-380. doi: 10.1093/jxb/ert453

Sunkar, R., Girke, T., Jain, P. K., and Zhu, J. K. (2005). Cloning and characterization of microRNAs from rice. Plant Cell 17, 1397-1411. doi: 10.1105/tpc.105.031682

Tang, G. (2010). Plant microRNAs: an insight into their gene structures and evolution. Semin. Cell Dev. Biol. 21, 782-789. doi: 10.1016/j.semcdb.2010.07.009

Taylor, R. S., Tarver, J. E., Hiscock, S. J., and Donoghue, P. C. (2014). Evolutionary history of plant microRNAs. Trends Plant Sci. 19, 175-182. doi: 10.1016/j.tplants.2013.11.008

Vazquez, F., Blevins, T., Ailhas, J., Boller, T., and Meins, F. Jr. (2008). Evolution of Arabidopsis MIR genes generates novel microRNA classes. Nucleic Acids Res. 36, 6429-6438. doi: 10.1093/nar/gkn670

Voinnet, O. (2009). Origin, biogenesis, and activity of plant microRNAs. Cell 136, 669-687. doi: 10.1016/j.cell.2009.01.046

Wang, Y., Itaya, A., Zhong, X., Wu, Y., Zhang, J., van der Knaap, E., et al. (2011). Function and evolution of a MicroRNA that regulates a $\mathrm{Ca}^{2+}$-ATPase and triggers the formation of phased small interfering RNAs in tomato reproductive growth. Plant Cell 23, 3185-3203. doi: 10.1105/tpc.111.088013

Wei, B., Cai, T., Zhang, R., Li, A., Huo, N., Li, S., et al. (2009). Novel microRNAs uncovered by deep sequencing of small RNA transcriptomes in bread wheat (Triticum aestivum L.) and Brachypodium distachyon (L.) Beauv. Funct. Integr. Genomics 9, 499-511. doi: 10.1007/s10142-009-0128-9

Winter, J., and Diederichs, S. (2011). Argonaute proteins regulate microRNA stability: increased microRNA abundance by Argonaute proteins is due to microRNA stabilization. RNA Biol. 8, 1149-1157. doi: 10.4161/rna.8.6.17665

Wu, G. (2013). Plant microRNAs and development. J. Genet. Genomics 40, 217-230. doi: 10.1016/j.jgg.2013.04.002

Wu, G., Park, M. Y., Conway, S. R., Wang, J. W., Weigel, D., and Poethig, R. S. (2009a). The sequential action of miR156 and miR172 regulates developmental timing in Arabidopsis. Cell 138, 750-759. doi: 10.1016/j.cell.2009.06.031

Wu, L., Zhang, Q., Zhou, H., Ni, F., Wu, X., and Qi, Y. (2009b). Rice microRNA effector complexes and targets. Plant Cell 21, 3421-3435. doi: 10.1105/tpc. 109.070938

Wu, L., Liu, D., Wu, J., Zhang, R., Qin, Z., Li, A., et al. (2013). Regulation of FLOWERING LOCUS T by a microRNA in Brachypodium distachyon. Plant Cell 25, 4363-4377. doi: 10.1105/tpc.113.118620
Wu, L., Mao, L., and Qi, Y. (2012). Roles of DICER-LIKE and ARGONAUTE proteins in TAS-derived small interfering RNA-triggered DNA methylation. Plant Physiol. 160, 990-999. doi: 10.1104/pp.112.200279

Wu, L., Zhou, H., Zhang, Q., Zhang, J., Ni, F., Liu, C., et al. (2010). DNA methylation mediated by a microRNA pathway. Mol. Cell 38, 465-475. doi: 10.1016/j.molcel.2010.03.008

Yan, K., Liu, P., Wu, C. A., Yang, G. D., Xu, R., Guo, Q. H., et al. (2012). Stress-induced alternative splicing provides a mechanism for the regulation of microRNA processing in Arabidopsis thaliana. Mol. Cell 48, 521-531. doi: 10.1016/j.molcel.2012.08.032

Yan, Y., Wang, H., Hamera, S., Chen, X., and Fang, R. (2014). miR444a has multiple functions in the rice nitrate-signaling pathway. Plant J. 78, 44-55. doi: $10.1111 /$ tpj. 12446

Yoshikawa, M., Iki, T., Tsutsui, Y., Miyashita, K., Poethig, R. S., Habu, Y., et al. (2013). 3' fragment of miR173-programmed RISC-cleaved RNA is protected from degradation in a complex with RISC and SGS3. Proc. Natl. Acad. Sci. U.S.A. 110, 4117-4122. doi: 10.1073/pnas.1217050110

Yu, B., Yang, Z., Li, J., Minakhina, S., Yang, M., Padgett, R. W., et al. (2005). Methylation as a crucial step in plant microRNA biogenesis. Science 307, 932935. doi: 10.1126/science.1107130

Zhai, J., Jeong, D. H., De Paoli, E., Park, S., Rosen, B. D., Li, Y., et al. (2011). MicroRNAs as master regulators of the plant NB-LRR defense gene family via the production of phased, trans-acting siRNAs. Genes Dev. 25, 2540-2553. doi: 10.1101/gad.177527.111

Zhang, J., Xu, Y., Huan, Q., and Chong, K. (2009). Deep sequencing of Brachypodium small RNAs at the global genome level identifies microRNAs involved in cold stress response. BMC Genomics 10:449. doi: 10.1186/1471-2164$10-449$

Zhang, Y. C., Yu, Y., Wang, C. Y., Li, Z. Y., Liu, Q., Xu, J., et al. (2013). Overexpression of microRNA OsmiR397 improves rice yield by increasing grain size and promoting panicle branching. Nat. Biotechnol. 31, 848-852. doi: $10.1038 /$ nbt.2646

Zhao, T., Li, G., Mi, S., Li, S., Hannon, G. J., Wang, X. J., et al. (2007). A complex system of small RNAs in the unicellular green alga Chlamydomonas reinhardtii. Genes Dev. 21, 1190-1203. doi: 10.1101/gad.1543507

Conflict of Interest Statement: The authors declare that the research was conducted in the absence of any commercial or financial relationships that could be construed as a potential conflict of interest.

Received: 20 September 2014; paper pending published: 30 September 2014; accepted: 09 October 2014; published online: 28 October 2014.

Citation: Qin Z, Li C, Mao L and Wu L (2014) Novel insights from nonconserved microRNAs in plants. Front. Plant Sci. 5:586. doi: 10.3389/fpls.2014. 00586

This article was submitted to Plant Genetics and Genomics, a section of the journal Frontiers in Plant Science.

Copyright $\odot 2014$ Qin, Li, Mao and Wu. This is an open-access article distributed under the terms of the Creative Commons Attribution License (CC BY). The use, distribution or reproduction in other forums is permitted, provided the original author(s) or licensor are credited and that the original publication in this journal is cited, in accordance with accepted academic practice. No use, distribution or reproduction is permitted which does not comply with these terms. 